\title{
EDITORIAL \\ Multiple myeloma presenting as an unknown primary disease: to operate or not to operate, that is the question
}

\author{
Mark H. Bilsky, MD, and Ori Barzilai, MD \\ Department of Neurosurgery, Memorial Sloan Kettering Cancer Center, New York, New York
}

$\mathrm{B}$ URKS et al. report a series of 43 patients presenting to the emergency room (ER) with newly diagnosed spine tumors of unknown primary origin who required decompression and instrumentation for high-grade epidural spinal cord compression (ESCC) and/or an unstable fracture. ${ }^{1}$ Overall, $51 \%$ were ultimately diagnosed with multiple myeloma (MM) or plasmacytoma, and 77\% presented with a neurological deficit and Bilsky ESCC score 2 or 3 . Despite the majority of unknown tumors in this series ultimately being diagnosed with radiation- and chemotherapy-sensitive plasma cell dyscrasias, the authors very appropriately took these patients expeditiously to surgery for decompression and instrumented fixation. Urgent or emergency surgery in the absence of a diagnosis prevents catastrophic neurological decompensation or ongoing instability pain while awaiting definitive pathology findings from a needle biopsy. In patients with neurological symptoms or high-grade ESCC with an unknown primary tumor, this management strategy is critically important for providers to recognize and adopt.

The experience garnered at the University of Miami ER mirrors our own experience at a tertiary cancer center where many patients presenting with spine fractures and high-grade ESCC in the absence of a known precedent cancer ultimately receive a diagnosis of MM or plasmacytoma. MM with spine involvement often presents as an emergency for two reasons, as follows. 1) Back pain is ubiquitous in the general population. Patients with MM will often give a history of experiencing months of biological back pain reflective of inflammation that is common not only in tumors, but also in virtually every benign spine pathology. In the absence of a history of cancer or known metastatic disease, patients with back pain are traditionally managed conservatively for an extended period prior to imaging - thus delaying the diagnosis. 2) MM is uniquely osteolytic, not only due to osteoclast activation via increased expression of RANKL and decreased expres- sion of osteoprotegerin, but also secondary to osteoblast inhibition by activation of the $W N T$ pathway, purportedly by plasma cell secretion of Dickkopf-1. ${ }^{2}$ For these reasons the patient's ER visit is often prompted by mechanical back pain and neurological issues resulting from an unstable pathological burst fracture and consequent high-grade ESCC and myelopathy.

Predicated on the neurological, oncological, mechanical, and systemic (NOMS) framework, ${ }^{3}$ patients with a known diagnosis of MM can often be treated with radiation or chemotherapy even in the setting of high-grade ESCC and myelopathy. Additionally, instability can typically be managed with percutaneous cement augmentation (i.e., kyphoplasty or vertebroplasty) and/or percutaneous pedicle screws. Fortunately, open surgery is therefore rarely indicated because it is complicated in MM by two factors: 1) hypervascularity of the tumor that is not amenable to embolization; and 2) difficulty in holding instrumented fixation due to tumor- and steroid-induced osteoporosis. The recent introduction of fenestrated polymethylmethacrylate (PMMA)-augmented pedicle screw fixation is a major advance in the treatment of tumors such as MM, and is now routinely used in our institution for percutaneous and open surgery. ${ }^{4-6}$

In the paper by Burks et al., ${ }^{1}$ they describe the major remaining indication for open spine surgery in the treatment of MM; i.e., a burst fracture with high-grade ESCC and myelopathy in which the diagnosis is unknown at presentation. Establishing a definitive diagnosis with a needle biopsy or a presumptive diagnosis with serum protein electrophoresis can take days to weeks. Before we adopted a more aggressive stance 20 years ago, delayed treatment while awaiting a diagnosis often led to acute catastrophic neurological decline even when temporized by high-dose steroids. Therefore, in the setting of an unknown diagnosis with high-grade ESCC and an unstable fracture, the unknown tumor should be presumed to be radioresistant 
and the patient should be taken to urgent or emergency surgery unless he or she has major medical contraindications. Surgery provides immediate decompression that optimizes the chance for improving neurological function and recovery of ambulation regardless of the radiosensitivity of the tumor, as well as providing instrumented stabilization. If ultimately the operative biopsy confirms $\mathrm{MM}$, radiation and/or chemotherapy are very effective as postoperative adjuvants to achieve both local and systemic tumor control.

In addition to MM, primary bone tumors are the other class of tumors that present to the ER with myelopathy resulting from high-grade ESCC in the absence of known diagnosis. Mobile-spine chordomas often present with this scenario. Some spine and oncology surgeons have advocated that all tumors should be biopsied, not only to avoid taking a patient with a radiosensitive tumor such as MM to the operating room, but also to avoid performing an intralesional resection rather than a planned en bloc excision for chordoma. One must recognize that chordoma is extremely rare (1 per million population), so there is a very low likelihood of a patient presenting with this scenario compared to MM or other metastases. Perhaps more importantly, patients with chordoma resulting in an unstable fracture and high-grade ESCC are not candidates for curative en bloc resection to achieve wide margins.

Recent evidence suggests that chordoma is very radiosensitive to stereotactic radiosurgery (SRS), conventionally fractionated proton beam therapy, and carbon ion therapy. In a series reported by Jin et al., 35 chordomas involving the mobile spine or sacrum were treated with SRS at a median dose of $24 \mathrm{~Gy}$ in a single fraction. ${ }^{7}$ In this cohort, 12 received SRS as definitive therapy, 12 underwent intralesional gross-total resection or separation surgery, and 11 received en bloc excision. Among the 32 patients receiving $24 \mathrm{~Gy}$ in a single fraction, the 3- and 5-year local recurrence-free survival rates were $96.3 \%$ and $89.9 \%$, respectively. The extent of surgery was not associated with local recurrence-free survival. In other words, intralesional surgery for chordoma to preserve or improve neurological function can still be salvaged with SRS to achieve local tumor control.

The critically important concept of immediate decompression and fixation in the setting of high-grade ESCC and instability in a patient without a known cancer is demonstrated in this superb series from the University of Mi- ami. Even if the tumor is MM or conversely, chordoma, the surgeon's obligation is to get the patient out of trouble and then to meaningful radiation and/or chemotherapy. In principle, delaying effective surgical therapy to establish a radiosensitive diagnosis or conversely, a tumor traditionally requiring en bloc excision, jeopardizes neurological function and does not impact local tumor control. Urgent or emergency surgery is the right call in this population.

https://thejns.org/doi/abs/10.3171/2021.2.FOCUS21108

\section{References}

1. Burks JD, Elarjani T, Jamshidi AM, et al. Vertebral multiple myeloma with pathological fracture: the most common etiology for emergency spine surgery in patients with no cancer diagnosis on admission. Neurosurg Focus. 2021;50(5):E2.

2. Terpos E, Sezer O, Croucher P, Dimopoulos MA. Myeloma bone disease and proteasome inhibition therapies. Blood. 2007;110(4):1098-1104.

3. Barzilai O, Laufer I, Yamada Y, et al. Integrating evidencebased medicine for treatment of spinal metastases into a decision framework: neurologic, oncologic, mechanical stability and systemic disease. J Clin Oncol. 2017;35(21): 2419-2427.

4. Barzilai O, McLaughlin L, Lis E, et al. Utility of cement augmentation via percutaneous fenestrated pedicle screws for stabilization of cancer-related spinal instability. Oper Neurosurg (Hagerstown). 2019;16(5):595-599.

5. Moussazadeh N, Rubin DG, McLaughlin L, et al. Shortsegment percutaneous pedicle screw fixation with cement augmentation for tumor-induced spinal instability. Spine J. 2015;15(7):1609-1617.

6. Newman WC, Amin AG, Villavieja J, et al. Short-segment cement-augmented fixation in open separation surgery of metastatic epidural spinal cord compression: initial experience. Neurosurg Focus. 2021;50(5):E11.

7. Jin CJ, Berry-Candelario J, Reiner AS, et al. Long-term outcomes of high-dose single-fraction radiosurgery for chordomas of the spine and sacrum. J Neurosurg Spine. 2020; 32(1):79-88.

\section{Disclosures}

The authors report no conflict of interest.

\section{Correspondence}

Mark H. Bilsky: bilskym@mskcc.org.

\section{INCLUDE WHEN CITING}

DOI: 10.3171/2021.2.FOCUS21108. 